\title{
HELIUM SHELL-FLASHES IN ACCRETING WHITE DWARFS
}

\author{
M. Y. Fujimoto \\ Takada Branch, Faculty of Education, Niigata University \\ and \\ D. Sugimoto \\ Department of Earth Science and Astronomy \\ College of General Education, University of Tokyo
}

When gas is accreted onto a carbon-oxygen white dwarf, a hydrogen shell-flash is triggered. Recently such phenomena are studied by many authors in relation to nova explosions and rekindling of white dwarfs. Unless all of the accreted gas is ejected by the process of the nova, a helium zone is formed as a result of hydrogen she11-burning. As the hydrogen shell-flashes recur many times, the helium zone grows gradually in mass. Then the helium shell-flash will be ignited as in the deep interior of ordinary red giant stars. We have investigated such process,simulating it by helium accretion at a constant rate. In the present paper we show that the helium shell-flashes result in a variety of strengths depending upon situations.

According to Sugimoto and Fujimoto (1978) and Fujimoto and Sugimoto (1979), the strength of the helium shell-flash depends on the degree of cooling in the helium zone. As it is cooled more before the ignition, the resultant shell-flash is stronger. There are two parameters in our problems in relation to the cooling. One is how long a time it has been cooled before the onset of the accretion, which we shall call the precooling time, $t_{p}$. It is characterized also by the intrinsic luminosity $I_{\text {pl }}$ of the white dwarf before the accretion. Another parameter is the accretion rate $\mathrm{dM}_{1} / \mathrm{dt}$. They are summarized in Tables 1 and 2 . Those models are constructed from the red giant stars whose core mass was $M_{1}=1.0766 M_{0}$. Case $A$ represents a hot white dwarf and Case $B$ a cool one. In Case $A$ we added a thin hydrogen-rich envelope as will be discussed later.

For different accretion rates we computed evolutions of accreting white dwarfs starting from the same initial model B. Results of computation are summarized in Table 2. If the accretion is fast (Case Bl, B2 and B3), the temperature of the helium zone rises by the compression as seen in Figure 1, where evolutionary loci of the bottom of the he.lium zone are shown in the density-temperature diagram. When the accretion rate is higher, the increase in the temperature is steeper, and the helium shell-flash is triggered with a smaller mass $\Delta M_{\mathrm{He}}$ accreted. 
TABLE 1. Initial Models

\begin{tabular}{cccccc}
\hline & $t_{\mathrm{p}}(\mathrm{Yr})$ & $\mathrm{L}_{\mathrm{ph}} / \mathrm{L}_{\odot}$ & $\log \mathrm{T}_{\mathrm{eff}}(\mathrm{K})$ & $\left.\mathrm{M}_{\mathrm{H}} / \mathrm{M}_{\odot}{ }^{\star}\right)$ & $\mathrm{M}_{\mathrm{He}} / \mathrm{M}_{\odot}$ \\
\hline case A & $3.7 \times 10^{3}$ & $1.62 \times 10^{2}$ & 5.32 & $4.2 \times 10^{-6}$ & $2.2 \times 10^{-6}$ \\
case B & $9.6 \times 10^{7}$ & $2.0 \times 10^{-2}$ & 4.40 & - & $7.9 \times 10^{-5}$ \\
\hline
\end{tabular}

*) The mass of the hydrogen-rich envelope

TABLE 2. Models of Accretion

\begin{tabular}{lccccc}
\hline \multicolumn{1}{c}{ Case } & \multicolumn{1}{c}{$\mathrm{Al}$} & $\mathrm{B} 1$ & $\mathrm{~B} 2$ & $\mathrm{~B} 3$ & $\mathrm{~B} 4$ \\
\hline $\mathrm{dM}_{1} / \mathrm{dt}\left(\mathrm{M}_{\odot} \mathrm{Yr}^{-1}\right)$ & $3.16 \times 10^{-8}$ & $1.71 \times 10^{-7}$ & $3.55 \times 10^{-8}$ & $3.16 \times 10^{-9}$ & $3.16 \times 10^{-10}$ \\
duration $(\mathrm{Yr})$ & $1.12 \times 10^{5}$ & $8.6 \times 10^{4}$ & $2.7 \times 10^{6}$ & $9.1 \times 10^{7}$ & $1.02 \times 10^{9}$ \\
$\Delta \mathrm{M}_{\mathrm{H}} / \mathrm{M}_{\odot}$ & $3.3 \times 10^{-6}$ & - & - & - & - \\
$\Delta \mathrm{M}_{\mathrm{He}} / \mathrm{M}_{\odot}$ & $3.5 \times 10^{-3}$ & $1.48 \times 10^{-2}$ & 0.097 & 0.29 & 0.32 \\
$\Delta \mathrm{M}_{2} / \mathrm{M}_{\odot}$ & $1.89 \times 10^{-3}$ & $3.7 \times 10^{-3}$ & 0.043 & 0.147 & - \\
\hline
\end{tabular}

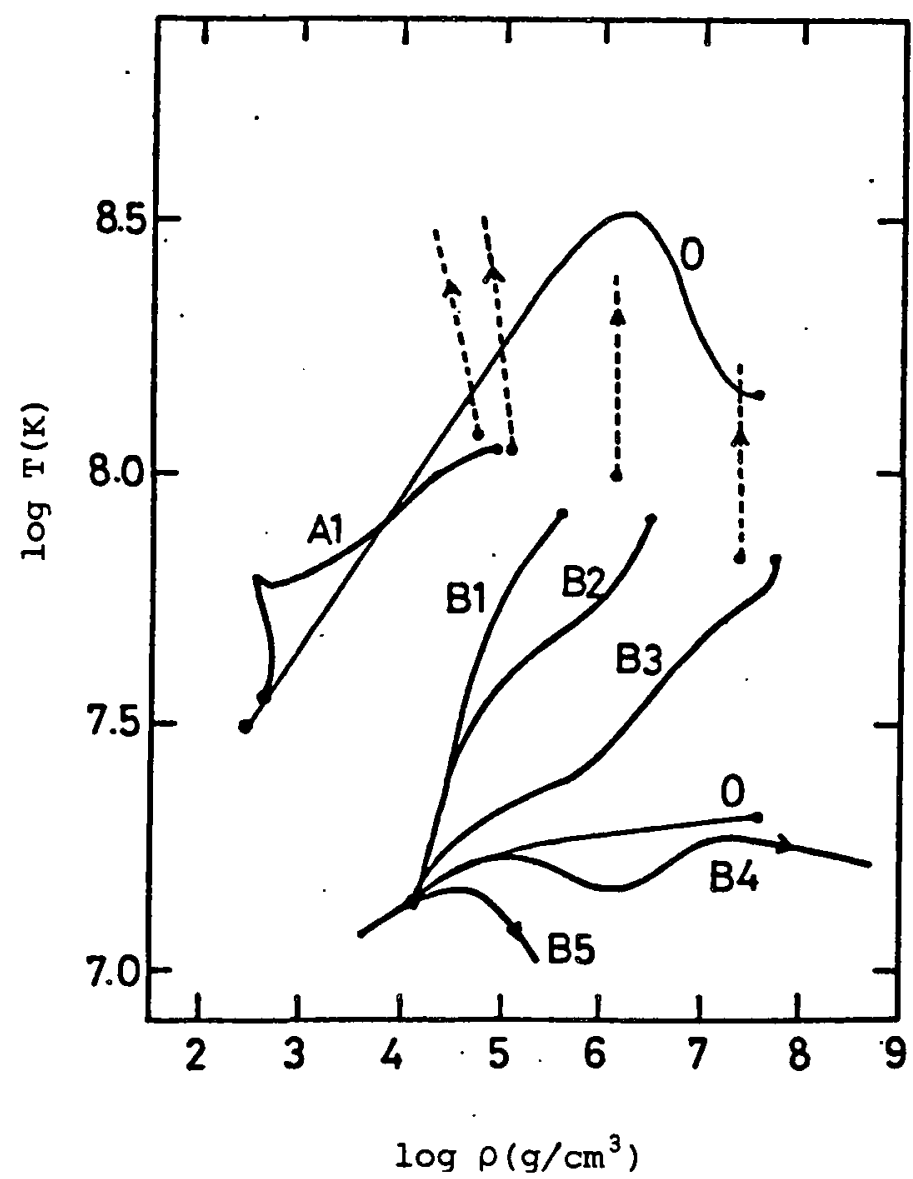

Fig. 1

Evolutionary loci of the bottom of helium zone in the densitytemperature diagram (thick solid curves). Dashed curves represent the helium burningshell. Thin solid curves are structure lines for initial models (attached with 0 ). 
Thus the values of $\Delta \mathrm{M}_{\mathrm{He}}$ at the ignition points are different by a factor of about twenty between Cases BI and B3, for example.

On the contrary, when the accretion rate is lower than for case B4, the temperature increases more slowly or even decreases as in Case $B 5$ for which the accretion rate is as low as $\mathrm{dM}_{1} / \mathrm{dt}=3.16 \times 10^{-1} \mathrm{M}_{\mathrm{O} Y \mathrm{r}^{-1}}$. However, in these cases of very slow accretion, helium is ignited by pycnonuclear reaction when the density reaches $3 \times 10^{8} \mathrm{~g} / \mathrm{cm}^{3}$ (Mazurek, 1973). When the concentration of ${ }^{14} \mathrm{~N}$ is appreciable, reactions

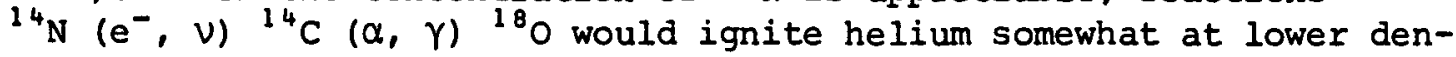
sity (Kaminishi, Arai, and Yoshinaga, 1975).

For Case $A$ of a hot white dwarf, helium is ignited at the density smaller than that for Case Bl in spite of lower accretion rate. The reason is that the specific entropy of the initial model is high.

We computed the helium shell-flash through its peak for Cases AI and B2. In any cases the shell-flash is triggered in a layer which lies above the bottom of the helium zone. The mass contained above the helium burning-shell $\Delta \mathrm{M}_{2}$ is 2-4 times smaller than $\Delta \mathrm{M}_{\mathrm{He}}$. The strength of the resultant peak is higher for larger $\Delta \mathrm{M}_{2}$ (Sugimoto and Fujimoto, 1978), and the maximum energy generations amount to $\mathrm{L}_{\mathrm{He}} \mathrm{max}^{\max }=$ $2.8 \times 10^{9} \mathrm{~L}_{\odot}$ and $8.4 \times 10^{15} \mathrm{~L}_{\odot}$ for cases $\mathrm{Al}$ and $\mathrm{B} 2$, respectively. Changes in the structure lines are shown in Figures 2 and 3 , for Case $B 2$ and $A 1$ respectively.

In Case B 2, the temperature gradient becomes very steep and superadiabatic as seen in the structure line at stage \#2. This implies that the heat cannot be transported by convection any more. Due to this blocking of heat, the timescale of expansion $d t / d n_{p}$ in the burning shell becomes even smaller than the free-fall timescale. If the inertia term had been taken into account in the equation of hydrodynamics, it would have been well dynamic and a detonation wave would have been formed (Nomoto and Sugimoto, 1977). The available energy is as much as $\Delta M_{\mathrm{He}} \times \mathrm{E}_{\mathrm{He}} \gtrsim 10^{50} \mathrm{erg}$, and explosion would be strong enough for a Type I supernova. Its remnant could be a white dwarf.

In the discussion above we neglected the hydrogen-rich envelope. However, it is important in relation to $s$-process nucleosynthesis. Therefore, we attached a hydrogen-rich envelope for models of $\mathrm{Al}$ as seen in Table 1. Even in Case $\mathrm{Al}, \mathrm{L}_{\mathrm{He}} \mathrm{max}^{\max }$ three hundred times stronger than ordinary shell-flashes in the red giant stars. As seen in Figure 3, the convection in the helium zone extended outward (\#1) and then reached the bottom of the hydrogen-rich envelope (\#2). Therefore, protons will be mixed into the helium zone and produce neutrons via ${ }^{12} C(p, \gamma){ }^{13} \mathrm{~N}\left(e^{+} \nu\right){ }^{13} \mathrm{C}(\alpha, n){ }^{16} \mathrm{O}$. These neutrons will be absorbed by iron group nuclei to synthesize the s-process elements. It is to be noticed that the cause of such mixing is not ascribed to the strength of the flash but to a lower entropy in such a thin hydrogen-rich envelope.

During the declining phase of the flash, the helium zone and the hydrogen-rich envelope expand greatly (stage \#3). As the effective temperature decreases the surface convection zone becomes deeper and 


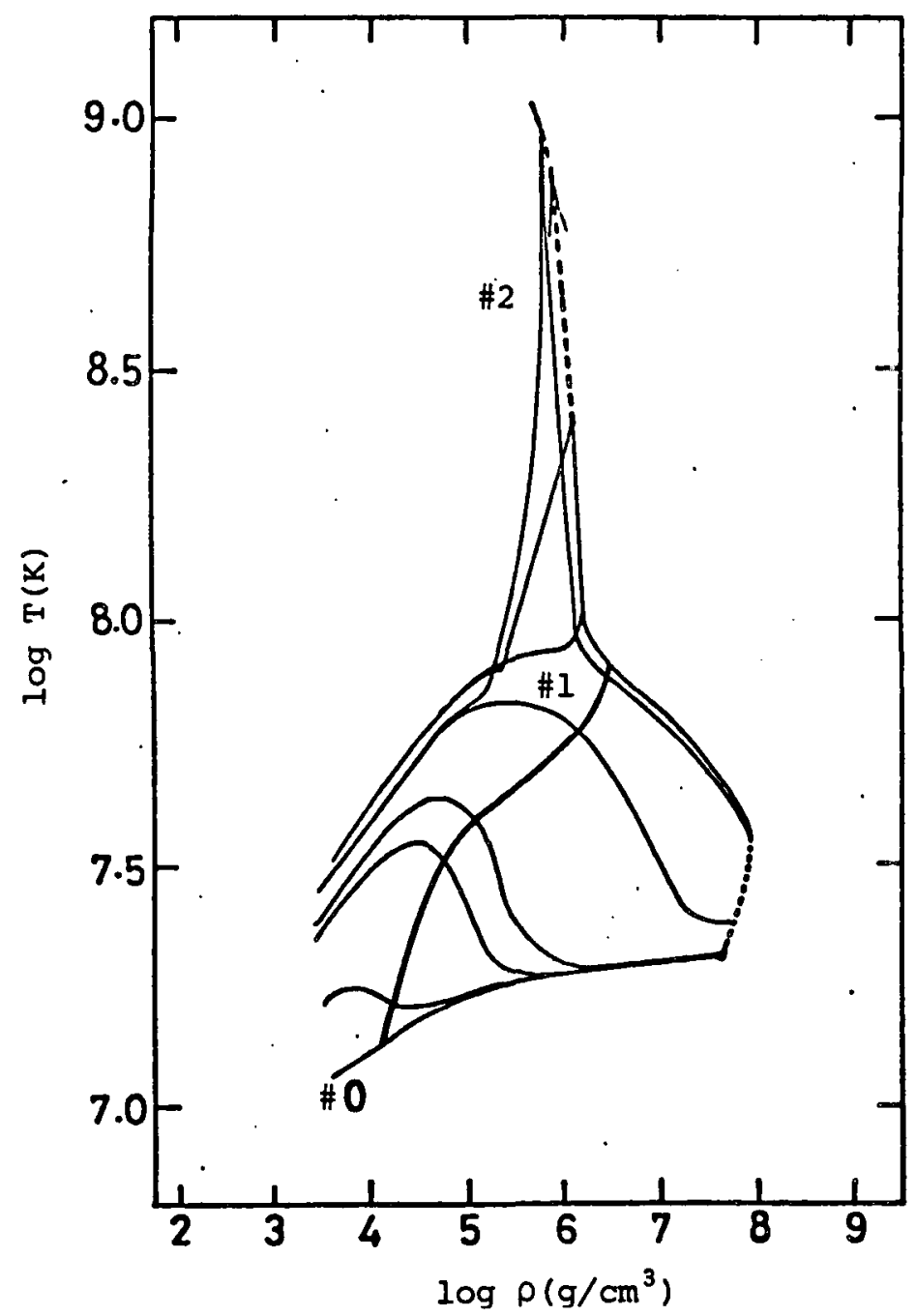

Fig. 2

Changes of structure

lines during the shell-

flash for Case B2. The initial model, the ignition of the helium shellflash, and the peak of the nuclear energy generation are denoted by the stage numbers $\# 0, \# 1$, and \#2, respectively. The dashed and dotted lines represent the loci of helium-burning shell and the center, respectively. The thick solid line is the evolutionary loci of the bottom of the helium zone.

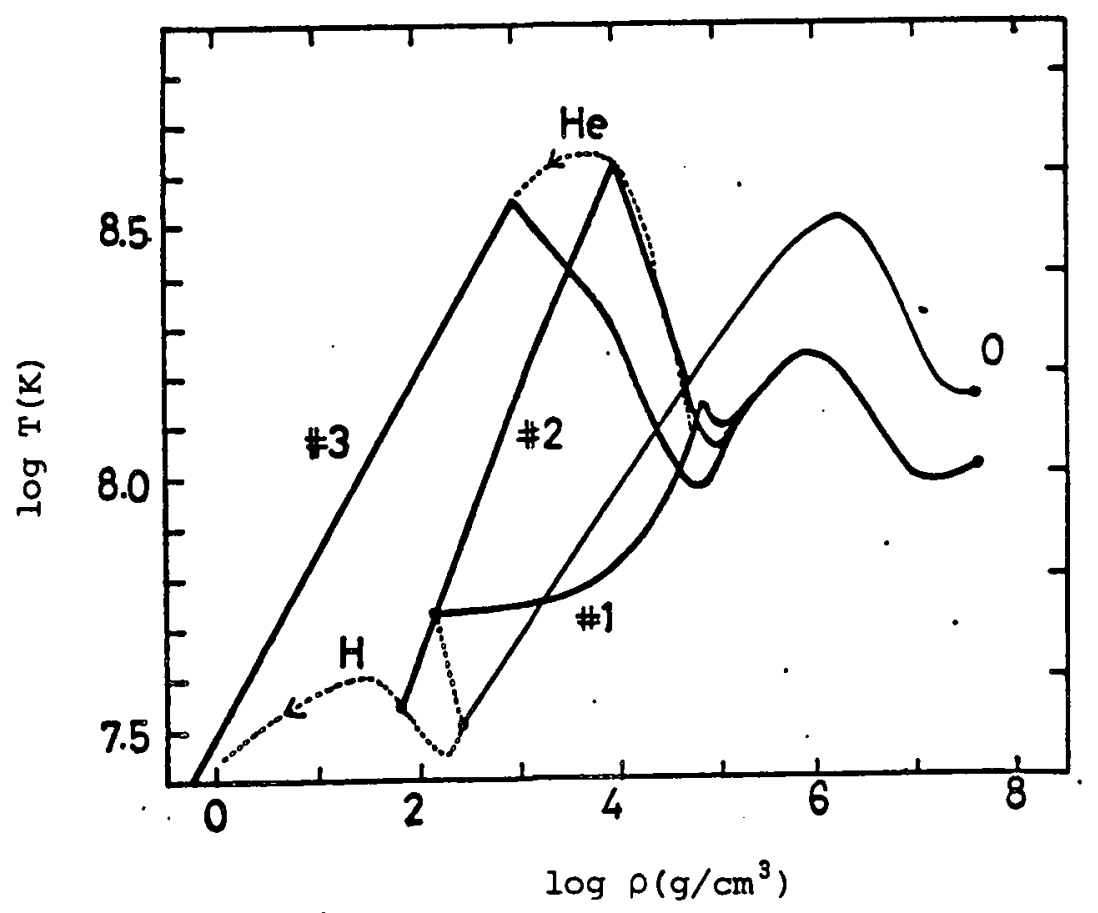

Fig. 3

The same as for Fig. 2, but for Case Al. The dotted curves represent the helium-burning shell (He) and the bottom of the hydrogen-rich envelope $(H)$. stage \#3 is th: declining phase of the shellflash. 
begins to dredge up the products of the nucleosynthesis. Such stars may be observed as hydrogen deficient carbon stars. If these are the case, the Type I supernovae could be related with hydrogen deficient carbon stars as discussed by Wheeler (1978) from the stand-point of statistics.

\section{REFERENCES}

Fujimoto, M.Y., and Sugimoto, D. 1979, Publ. Astron. Soc, Japan, 31, 1. Kaminishi, K., Arai, K., and Yoshinaga, K. 1975, Prog. Theor. Phys., Kyoto, 53, 1855 . Mazurek, T.J. 1973, Astrophys. Space. Sci. 23, 365.

Nomoto, K., and Sugimoto, D., 1977, Publ. Astron. Soc. Japan, 29, 765. Sugimoto, D., and Fujimoto, M.Y. 1978, Publ. Astron. Soc. Japan, 30 , 467.

Wheeler, J.C. 1978 in Proceedings of the Workshop on Supernovae and Supernova Remnants held in Erice. 